American J. of Engineering and Applied Sciences 4 (4): 440-447, 2011

ISSN 1941-7020

(C) 2014 W. Choi et al., This open access article is distributed under a Creative Commons Attribution

(CC-BY) 3.0 license

\title{
Detection of Subsurface Defects in Concrete Bridge Deck Joints
}

\author{
${ }^{1}$ Wonchang Choi, ${ }^{2}$ Larry Rickard, ${ }^{1}$ Taher Abu-Lebdeh and ${ }^{1}$ Miguel Picornell \\ ${ }^{1}$ Department of Civil Architectural and Environmental Engineering, \\ North Carolina A and T State University, Greensboro, 27411, NC, USA \\ ${ }^{2}$ Department of Transportation, North Carolina, Greensboro, 27411, NC, USA
}

\begin{abstract}
Problem statement: The integrity of deck joints in highway bridges plays a major role to determine overall performance of bridge system. As the bridge maintenance program, the defects in deck joints have historically been detected by conventional non-destructive testing and evaluation methods such as visual inspection, chain-dragging and by the detecting sounds under the traffic. Future bridge maintenance challenges will demand the development of techniques and procedures to detect and monitor such defects before they become apparent. Approach: Two non-destructive methods; namely Ground Penetration Radar (GPR) and Seismic Properties Analyzer (SPA) were employed to assess the integrity of deck joins installed in North Carolina bridges. Results: The results obtained with the GPR and SPA allows to quantify the subsurface defects in bridge deck joints. Conclusion: The practical application and limitations of each method are discussed in this study.
\end{abstract}

Key words: Ground penetration radar, seismic properties analyzer, de-bonding, Association of State Highway Transportation Officials (AASHTO), Seismic Properties Analyzer (SPA), Ground Penetration Radar (GPR), Visual Inspection (VI), Impact-Echo (IE)

\section{INTRODUCTION}

There are many factors which can affect the overall performance and longevity of highway bridges, including the integrity of its deck joints. They do not generally constitute a major portion of a bridge's construction cost, yet over time, joints that are improperly designed, installed or poorly maintained can cause damage that far exceeds their relative size and initial cost (Scotta et al., 2003). This possibility has become a concern with many states in the United States which has experienced problems with the bonding in the armored deck joints installed on many of its bridges.

Bettigole and Robison (1997) presented that American Association of State Highway Transportation Officials (AASHTO) believe that adequate expansion joints should:

- Accommodate all structural movement

- Possess sufficient load capacity

- Possess good riding characteristics

- Not present a hazard to traffic of all types

- Not place unnecessary stresses on the structure

- Not vibrate and be relatively quiet

- Be corrosion-resistant

Corresponding Author: Taher Abu-Lebdeh, Department of Civil Architectural and Environmental Engineering, North Carolina A and T State University, 1601 E. Market Street, Greensboro, 27411, NC, USA Tell: 336 334-7737 Fax: (336) 334-7126
- Allow for maintenance

- Protect the structure below it by restricting leakage

- Be reliable throughout the range of temperatures expected in service

In addition, deck joints should not impede or be damaged by snowplowing operations and should employ an anchorage system that supports the deck surface in its immediate vicinity (Xanthakos, 1996).

\section{MATERIALS AND METHODS}

An extensive deterioration was caused by apparent chloride intrusion due to seepage through the original joints. Some of this seepage undoubtedly occurred during the early life of the structure when its original bare concrete wearing surface was still exposed. As that surface deteriorated, at least one layer of asphalt concrete was applied. When fresh, this additional cover helped seal the original deck joints but decades of movement, freeze-thaw cycles. Caused cracks to appear, allowing further seepage.

These defects have historically been detected using conventional Non-Destructive Testing and Evaluation (NDT/E) methods, which generally include Visual 0 
Inspection (VI), chain-dragging, hammer blows and detecting the sounds made by traffic passing over suspect joints. The problem common to these techniques is that damage to the joint in question is usually severe enough to warrant its replacement by the time such methods are effective.

The use of advanced NDT/E technology - GroundPenetrating Radar (GPR) and Acoustic methods such as Impact-Echo (IE) and Surface Wave Seismic Analysis (SWSA) in particular is one possible solution to this problem. Their effectiveness in collecting quantitative data on de-lamination in bridge decks has been well established through decades of study and field use and has been repeatedly verified with ground truth data (Maser and Bernhardt, 2000). In the majority of study, the focus has been on whole deck assessment and not the examination of any particular area. There were several reasons for this, but the ones common to most of the technologies used were bulky equipment and the lack of real-time data display. NDT/E technology is advancing rapidly, however.

The current generation of portable equipment is relatively inexpensive, exhibits improved diagnostic capabilities and is easily deployed by a single operator. They are particularly well-suited to studies of highly localized areas such as deck joints. All of these attributes make these newer technologies an attractive alternative to the conventional traditional NDT/E methods that are normally used during routine bridge inspections.

This research will investigate the extension of the use of handheld GPR units and the Portable Seismic Properties Analyzer to the detection of subsurface defects and anomalies in and around bridge deck armor. Particular attention will be paid to exploring the challenges peculiar to these techniques with regard to armored deck joints and to their potential as an alternative or adjunct to conventional non-destructive testing techniques. In addition, study will attempt to quantify the results found and to study the possible feasibility of their incorporation into existing bridge maintenance programs.

NDT methods for detecting defects of deck joints: Ground Penetrating Radar (GPR): The use of GPR has been more common in various fields. Among them, the ASTM D6087 (2008) primarily describes the procedure for using GPR in the evaluation of asphaltcovered bridge decks. These methods are also valid for concrete decks or those with a concrete overlay, however. Procedures for the proper use and calibration of both air and ground-coupled GPR systems are listed. Also documented are two different algorithms for calculating the extent of any de-lamination present. One particularly noteworthy item is the attention paid to ensuring that passes made by the GPR unit are perpendicular to the top layer of reinforcing steel. One study that illustrates the effectiveness of GPR in the assessment of bridge decks was performed by the Arizona Department of Transportation (ADOT). That agency conducted a statewide survey that compared GPR results to those obtained by conventional NDT/E methods (Maser and Bernhardt, 2000). A total of 134 bridges were surveyed between December of 1998 and April of 1999. Attenuation of a given GPR signal is greater through concrete weakened by chloride intrusion or delaminating than in intact concrete. Therefore, deterioration in the bare concrete decks was detected by measuring the attenuation of the radar signal either through the entire thickness of the deck or through the concrete cover over the top layer of reinforcing steel. Attempts have been made to increase the accuracy of interpreting GPR data (Barnes et al., 2008).

The GPR unit used in this study is the Structures can $^{\mathrm{TM}}$ Mini (SSM), made by Geophysical Survey Systems, Inc. (GSSI) of Salem, New Hampshire. It is a compact, lightweight handheld unit designed expressly for the location of subsurface objects in concrete structures. The SSM works by calculating the relative differences between the dielectric constants in the material being scanned. The dielectric constant of a material is defined by GSSI as the ability of a material to hold an electric charge. A material's dielectric constant $(\varepsilon)$ is defined as follows in the Eq. 1:

$\varepsilon=\frac{\mathrm{C}}{\mathrm{C}_{\mathrm{o}}}$

where, Co is the capacitance between two parallel plates separated by a vacuum and $\mathrm{C}$ represents the capacitance between the same two parallel plates under identical conditions when separated by the dielectric material in question. Table 1 lists the dielectric constants for materials likely to be found in concrete structures (Young and Freedman, 1999).

According to the above table, air has a $\varepsilon$ of 1.0 for all intents and purposes; while water has a $\varepsilon$ of around 80.4 (the manual rounds this up to 81 ). Concrete has a nominal $\varepsilon$ of around 6 , depending on its age and environment. Because the SSM is optimized for subsurface analysis of concrete structures, however, it is necessary to identify $\varepsilon$ for concrete in different stages of curing. These are summarized in Table 2.

Table 1: Dielectric constant $\varepsilon$ for materials commonly found in concrete structure

\begin{tabular}{lllr}
\hline Material & $\varepsilon$ & Material & $\varepsilon$ \\
\hline Vacuum & 1.00000 & Polyvinyl Chloride (PVC) & 3.18 \\
Air (at 1 atm) & 1.00059 & Plexiglas & 3.40 \\
Teflon & 2.10000 & Glass & $5-10$ \\
Polyethylene & 2.25000 & Neoprene & 6.70 \\
Mica & $3-6000$ & Water & 80.40 \\
\hline
\end{tabular}


Table 2: Dielectric constant $\varepsilon$ for concrete in various stages of curing

\begin{tabular}{ll}
\hline Concrete age/environment & Approximate $\varepsilon$ \\
\hline Less than 2 months/wet environment & $9+$ \\
Less than 12 months/outside & $7-8$ \\
More than 12 months/dry & $5-6$
\end{tabular}
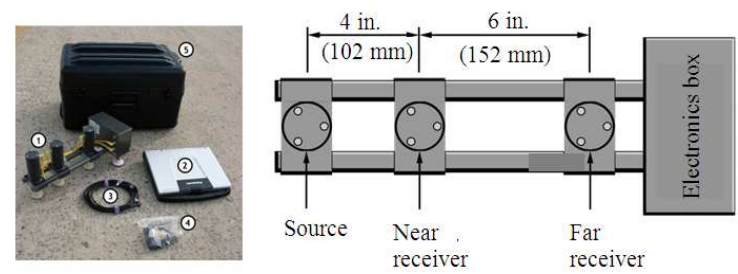

Fig. 1: General arrangement of the SPA

All of these materials will absorb the radio frequency energy produced by the scanner to some degree. As a result, the scanner's dominant colors will be Black-White-Black or White-Black-White, depending on whether the object beneath the surface has a $\varepsilon$ higher or lower than the surrounding concrete. This difference is called the Reflection coefficient (R) which is defined as follows in the Eq. 2:

$\mathrm{R}=\frac{\sqrt{\varepsilon_{2}}-\sqrt{\varepsilon_{1}}}{\sqrt{\varepsilon_{2}}+\sqrt{\varepsilon_{21}}}$

Ultrasonic Surface Waves (USW): One of the newer tools is the Seismic Pavement Analyzer (SPA) as shown in Fig. 1. This instrument combines the capabilities of Impact-Echo (IE) testing and Ultrasonic Surface Wave Seismic Analysis (USW) in one unit. Acoustic testing of concrete by electromechanical methods is currently outlined in two ASTM standards (ASTM C3183, 2010). The first (C1383-04) involves the measurement of $\mathrm{P}$ wave speed and concrete plate thickness. The second (ASTM C1740, 2010) outlines procedures for evaluating the actual condition of concrete plates.

The SPA was employed in one 2007 study to investigate deboning in concrete slabs on Texas Route 225 southwest of Houston (Celaya et al., 2007). Field records in the form of time records and frequency spectra were gathered; this data confirmed the shortcomings of using time-domain analysis of the reflected waves in IE testing. It also confirmed the long-standing use of the Fast Fourier Transform (FFT) and the effectiveness of frequency domain analysis in detecting marginally-delaminated slabs.

Another more recent involving the SPA was conducted in 2010. This study investigated the effectiveness of several different NDT methods in detecting deboning of hot-mix asphalt (HMA) layers in airport runways (Celaya et al., 2010). While this study did not specifically address issues with concrete bridge decks, it was nonetheless informative because the GPR and the SPA both proved effective despite the complications inherent to HMA as a test material.

The SPA used in this study is specifically designed to measure the dynamic modulus and overall quality of a variety of materials including concrete, asphalt, base materials and compacted soil. It works by repeatedly actuating the source, which generates a series of pulses in the material under test. The near and far receivers then receive these pulses after they have propagated through the test material. The accelerometers in the receivers then convert the received energy into an analog electrical signal. Conversion of this signal into a digital waveform takes place in the electronics box before being sent to the computer. Until this point, the signals produced by the SPA are in the time domain. Analysis of the material, however, requires their conversion into the frequency domain. This is done via the included software (SPA Manager), which performs a Discrete Fourier Transform. This develops the frequency signature and the dynamic modulus for the specific test point. The procedure outlined above requires that the SPA apparatus detect three distinct types of waveforms.

P-waves, also called Primary or Dilatational waves. These propagate horizontally and cause purely tensile stresses or "peaks" and compressive stresses or "troughs" in the material under test. Relative particle motion is back-and forth, parallel to the direction of propagation. $\mathrm{P}$ waves possess the fastest velocity of any of the three wave types outlined here; its theoretical velocity is dependent upon the material's elasticity and density as follows in the Eq. 3:

$$
\mathrm{V}_{\mathrm{P}}=\sqrt{\frac{\mathrm{E}(1-v)}{(1+v)(1-2 v) \rho}}
$$

\section{Where:}

$\mathrm{VP}=$ The theoretical $\mathrm{P}$ wave velocity

$\mathrm{E}=$ The material's modulus of elasticity

$v=$ Poisson's ratio and $\rho$ the material's density

The energy in this waveform is transferred by causing a ripple effect that is uniform at all levels of the material; any one particle within the material moves in a vertical line, causing shear within the material. Its theoretical velocity is determined by the Eq. 4:

$$
\mathrm{V}_{\mathrm{S}}=\sqrt{\frac{\mathrm{E}}{2(1+v) \rho}}
$$

where, Vs is the theoretical S wave velocity. 
R-waves, also known as Rayleigh or simply surface waves are primarily a surface waveform and are the results of $\mathrm{P}$ and $\mathrm{S}$ waves traveling near surface of a semi infinite body. The energy of the wave's propagation imparts both horizontal and vertical components to the motion of a given particle, which moves in a circular path. Where the " $\mathrm{R}$ " wave differs from the previous two is the fact that it is primarily a surface wave. Energy within the wave is not distributed evenly; relative particle motion decreases with depth in a linear fashion. The theoretical velocity of the Rayleigh wave is in the Eq. 5:

$\mathrm{V}_{\mathrm{R}}=\mathrm{V}_{\mathrm{S}} \frac{(0.87+1.12 \mathrm{v})}{(1+\mathrm{v})}$

where, VR is the theoretical $\mathrm{R}$ wave velocity.

The SPA uses R-waves to perform USW analysis. It determines the dynamic modulus of a given material by the Eq. 6 :

$\mathrm{E}=2 \rho(1+v)\left[\mathrm{V}_{\mathrm{R}}(1.13-0.16 v)\right]^{2}$

where, E is the material's dynamic modulus. Poisson's ratio $(v)$ for concrete typically falls between 0.15 and $0.20 ; 0.18$ will be assumed for this study. Likewise, the density of concrete $(\rho)$ will be assumed to be $150 \mathrm{Lb}$ $\mathrm{ft}^{-3}\left(2,402.8 \mathrm{~kg} \mathrm{~m}^{-3}\right)$.

Field evaluation: The finger joint on the Greyhound Court Bridge was typical of those used on NCDOT highway bridges built during the mid-twentieth century. It originally consisted of two interlocking steel plates, the wearing surfaces of which were manufactured with a diamond pattern to increase traction.

Because the condition of the deck joint itself was in question, the focus of the inspection shifted to the condition of that portion of the concrete deck to which the joint was bonded. This area was also scrutinized very closely for the same reasons listed above. Particular attention was paid to assessing the quality of the wearing surface because major defects in this area would also present difficulties when using the test equipment. The SSM, for example, requires that the surface under test be relatively uniform, because it is essentially a wheeled vehicle with very little vertical clearance.

Any major discontinuity (i.e. surface voids caused by spelling) could cause the unit to "bottom out", resulting in anomalous readings or damage to the unit. The quality of the surface in this area was found to be quite typical of the deck as a whole, exhibiting the weathering, map cracking and evidence of chloride intrusion noted in the report North Carolina Department of Transportation (Bridge No. 33017). There was very little spelling in this area and there was no visible evidence of damage. Tapping with a masonry hammer produced hollow sounds in some areas immediately adjacent to the joint, however. This was potential evidence of delaminating and tended to be more prominent toward the joint ends at the sidewalks, particularly the southern end.

\section{RESULTS}

Ground Penetration Radar (GPR): Data collection began after completion of the housekeeping tasks and initialization of the global settings. Before each scan set, a scan depth of 8 in. was chosen for several reasons, primarily because any delaminating or other phenomena connected to the bonding of the joint was unlikely to exist any deeper than the actual depth of the joint itself. Another reason was to avoid any possible reflection from deep layers of reinforcing steel or from any other metal, such as the corrugated metal decking used as forms on modern construction. Although the actual dielectric constant of the concrete was unknown, the Dielectric option was set to 6.1 considered to be a good estimate of the dielectric constant, $\varepsilon$ for fully cured concrete. The Rebar scans were performed next. The first of these was performed on the marked centerline (Fig. 2) since it intersected the upper layer of reinforcing steel at an angle of approximately 90.

As shown in Fig. 2, scanning began with the index point just over the interface and ended just beyond Station 25. The scan data was checked for completeness and accuracy before being stored. The procedure was then repeated to the left and right of the centerline at Offsets 22, 20 16, 12, 8 and 4. The Joint Scans were begun upon completion of the Rebar Scans. The procedure was used as that used for the Rebar Scans, except for the scan pattern. Stations to the right of the centerline were scanned first, followed by those on the left.

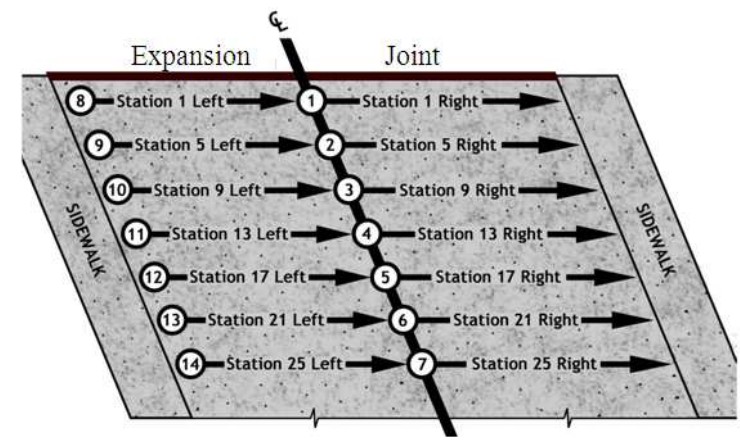

Fig. 2: Schematic of SSM scan sequence 
Am. J. Engg. \& Applied Sci., 4 (4): 440-447, 2011

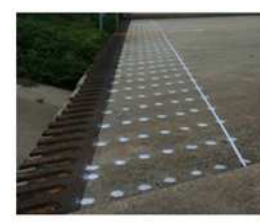

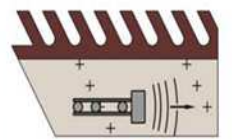

Offsets left

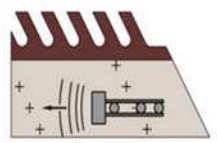

Offsets right
Fig. 3: SPA positions for left and right offsets

Seismic Properties Analyzer (SPA): Testing all 315 marked points in Fig. 3 would have required an absolute minimum of twelve $\mathrm{h}$, which was considered to be time and cost-prohibitive. Furthermore, real world testing on an intact structure would require that the procedure be performed on both sides of the joint, effectively doubling the test time. Therefore a decision was made to reduce the number of points to be tested. This was accomplished by limiting testing to the even offsets plus centerline point at stations 1, 9, 17 and 25 . This reduced the total number of test points N-92 and TT to approximately $31 / 2 \mathrm{~h}$. These numbers presented a much more realistic balance in terms of test time versus accurate representation of the concrete module surrounding the joint. One final detail needed to be decided before SPA testing could begin: the instrument's position over the point under test. The relationship between the IE and SWSA modes demonstrated that the data gathered for each required a different source-emitter combination. Therefore, the centerline of each test was necessarily different; the IE tests were centered between the source and near receiver, while the SWSA tests were centered between the source and far receiver.

Because the difference between the two was known and consistent, it was decided to position the SPA so that the centerline of the SWSA test mode lay directly over the point under test. Furthermore a decision was reached to face the SPA so that the source was positioned away from the centerline. This would further minimize the finite surface effects of concern.

The instrument was placed carefully over the first test point-Station 1, Offset 22 Left. The SPA completed three sets of measurements, after which the test data was automatically reduced. This data was reviewed for consistency before acceptance; any major variances in the waveform, SASW or IE graphs between the three individual measurements resulted in the instrument being repositioned over the test point and the measurements repeated. Otherwise, the SPA was placed over the next test point and the procedure repeated until data was collected on all 92 points. Complete results of the individual test points are provided in elsewhere (Rickard, 2011).

\section{DISCUSSION}

Ground Penetration Radar (GPR): A preliminary look at the scans in Fig. 4 revealed much about the bridge's structure. Scans closest to the joint under study revealed the relatively complex structure of the reinforcing steel in that area. The images also showed the bottom of the deck as well as the girder locations. Most noteworthy was the existence of several discolored or "ghosted" areas in each scan; these were considered possible evidence of delaminating. The next step in processing the data was the detection and quantification of any suspected delaminating. This was done visually by noting any extended areas of discoloration in each image that could not be accounted for by other objects such as reinforcing steel. A colorcoded system was devised based upon the four-tier system. Areas that exhibited little or no discoloration were not suspected to suffer from delaminating and were therefore left uncolored. Areas which showed a linear ghosting effect were considered to be slightly delaminated and were tinted green. Ghosted areas which tended to exhibit dark edges above and below were labeled as moderately delaminated and tinted yellow, while areas suspected to suffer from severe delaminating tended to exhibit rather well-defined dark boundaries; these were tinted red.

For the GPR testing, the ability to draw conclusions regarding the joint required that the scan data be superimposed on a map of the deck surface. Again, this was accomplished by using Micro station. Each area of suspected delaminating was drawn in its corresponding location on a scale outline of the joint area. The resulting delaminating map is illustrated in Fig. 5.

According to the GPR data, there is widespread evidence of damage to the concrete on the joint's left side. The most severe damage -those areas where deterioration was rated as moderate or severe - appears to have occurred in the immediate vicinity of the joint interface and in those areas toward the centerline. Apparent deterioration was less severe at the extreme left of the joint. The damage here was just as widespread, however and the area toward the gutter line still displayed some moderate to severe damage. The GPR data to the right of the centerline showed a similar pattern. With a few exceptions, the damage generally appears to be light toward the centerline and more severe toward the right side of the joint. The overall pattern of deterioration on this half of the joint appeared less dense, however. 
Am. J. Engg. \& Applied Sci., 4 (4): 440-447, 2011

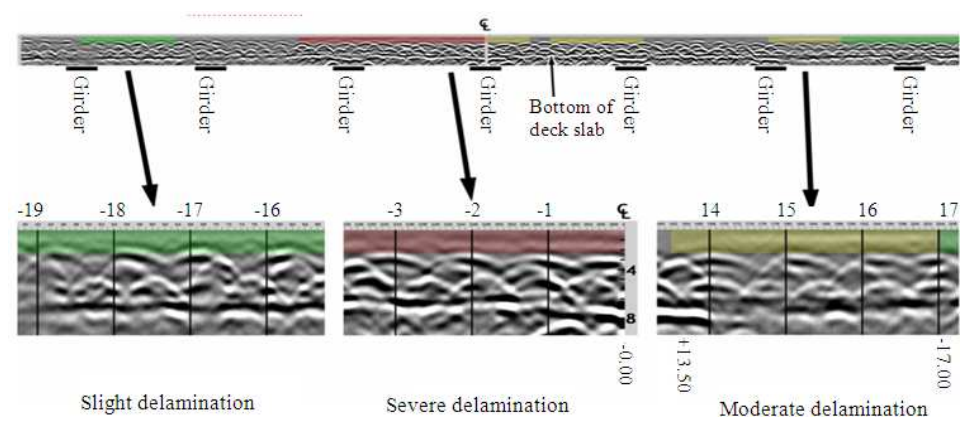

Fig. 4: Test result using GPR

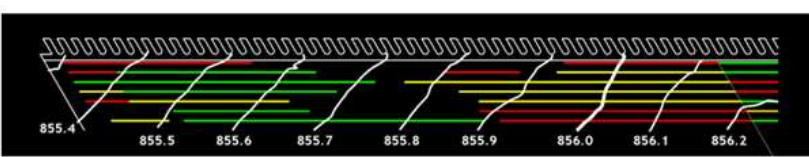

Offsets left

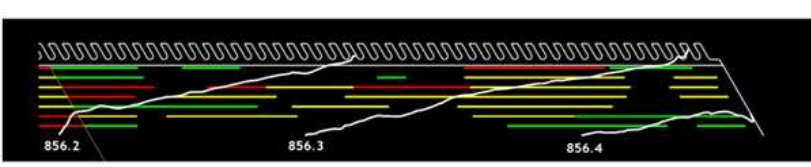

Offsets right

Light delamination $=$ Moderate delamination $\square$ Severe delamination GPR results

Fig. 5: Deterioration map: GPR data and surface survey results

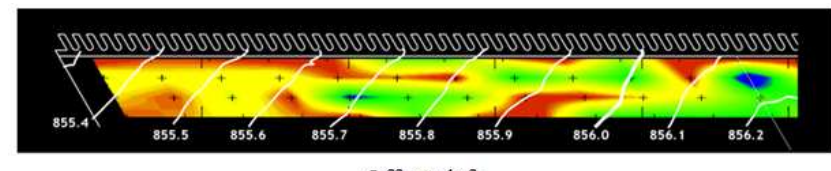

Offsets left

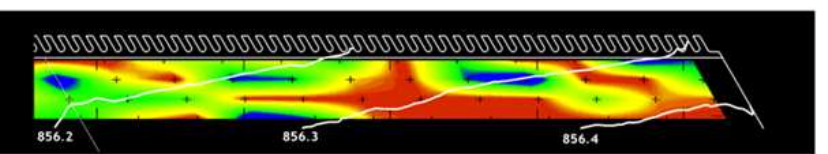

Offsets right

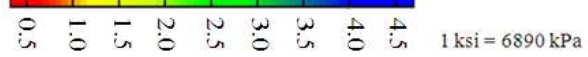

Dynamic modulus in $\left(10^{3} \mathrm{ksi}\right)$

Fig. 6: Deterioration map: SPA data and surface survey results

The majority of deterioration on both halves of the deck appears to lie in those areas directly in the vehicle wheel paths. This is evidenced by the relative scarcity of damage in the center of each lane. This fact correlates well with the outcome of a survey, where $100 \%$ of the respondents stated that damage to the armored joints was most apparent in these locations.
However, the widespread nature of the suspected deterioration as shown by the GPR data suggests that it may be due to causes other than deboning of the joint.

Seismic properties analyzer (SPA): The deterioration map based upon the SPA data is given in Fig. 6. According to this set of data, the majority of moderate 
to severe delaminating appear to lie in the area to the left of the centerline. The entire area approximately $7 \mathrm{ft}$. $(2.1 \mathrm{~m})$ to the right of the gutter appeared to be in relatively poor condition, with no reading over $2.0 \mathrm{ksi}$ (13.8 MPa). The most severe deterioration appeared at the joint interface and in several intermittent areas toward the centerline. The remainder of the readings showed module in the range of 2.0-3.0 ksi (13.8-20.7 $\mathrm{MPa})$, with only one reading over $3.5 \mathrm{ksi}(24.2 \mathrm{MPa})$.

Similar results were obtained with data from the right half of the joint. While there were more reading that indicated the presence of sound concrete (modulus $>3.5 \mathrm{ksi}(24.2 \mathrm{MPa}))$, the overall quality of the concrete was poor. There were areas of severe, moderate and slight deterioration detected in the immediate vicinity of the joint interface. A large area of severe deterioration was detected in the approximate center of the lane. No reading was obtained over $1.0 \mathrm{ksi}$ (6.89 $\mathrm{MPa})$ in this region, which extended from the joint interface to the testing limits.

Like the results gathered from the GPR data, the distribution and severity of the deterioration suggest that the damage in this vicinity was due to factors other than joint deboning. Unlike the GPR data, however, there seems to be no clear correlation between the damage as shown in the figure and vehicular wheel paths. Both of these appear to be supported by the IE data, which showed the deck surface to be in relatively poor condition throughout the test area.

\section{CONCLUSION}

This research focused on developing methods and techniques for detecting deboning and delaminating in armored bridge deck joints using portable NDT/E devices. The efficacy of this equipment in detecting defects in the concrete surrounding deck joints was also investigated. Observations and conclusions drawn from the gathered data are summarized as follows:

- $\quad$ The equipment for the GPR (SSM) and Acoustic methods (SPA) was quickly and easily deployed in the field, but the processing and mapping of the data was cumbersome and difficult

- The quality of the data gathered from all of the methods used GPR and Acoustic was highly dependent upon the quality of the surface under test. These methods may not be suitable for use on bridge decks where widespread delaminating or other deterioration is present

- In this study, GPR was very limited in its ability to detect delaminating or other anomalies below the first layer of reinforcing steel
- Of the methods used in this study, the SPA appeared to exhibit the greatest potential for detecting subsurface deterioration due to delaminating or deboning of deck joint armor

- Development of a reference system specific to the bridge or joint under test is crucial for the accurate mapping of gathered data. Such a system should account for factors such as deck width, curbing and skew

- Accurate mapping of data is the key to representing the overall condition of the joint bonding areas at the time of testing

- The effective use of NDT/E methods for limited areas (such as deck joints) is currently hampered by the nonexistence of a comprehensive mapping and evaluation system

\section{REFERENCES}

ASTM C1740, 2010. Standard practice for evaluating the condition of concrete plates using the impulseresponse method. ASTM. DOI: 10.1520/C1740-10

ASTM C3183, 2010. Standard Test Method for Measuring the P-Wave Speed and the Thickness of Concrete Plates Using the Impact-Echo Method. ASTM. DOI: 10.1520/C1383-04R10

ASTM D6087, 2008. Standard Test Method for Evaluating Asphalt-Covered Concrete Bridge Decks Using Ground Penetrating Radar. ASTM. DOI: $10.1520 / \mathrm{D} 6087-08$

Barnes, C.L., J.F. Trottierand D. Forgeron, 2008. Improved concrete bridge deck evaluation using GPR by accounting for signal depth-amplitude effects. NDT Int., 41: 427-433, DOI: 10.1016/j.ndteint.2008.03.005

Bettigole, N.H. and R. Robison, 1997. Bridge Decks: Design, Construction, Rehabilitation and Replacement. 1st Edn., ASCE Publications, New York, ISBN: 078440223X, pp: 118.

Celaya, M., P. Shokouhi and S. Nazarian, 2007. Assessment of debonding in concrete slabs using seismic methods. Trans. Res. Record J. Trans. Res. Board, 2016: 65-75. DOI: 10.3141/2016-08

Celaya, M., S. Nazarian, C. Rao and H.V. Quintus, 2010. Delamination detection of HMA airport pavements with NDT devices. FAA Worldwide Airport Technology Transfer Conference, Atlantic City. N.J., USA., pp: 1-16.

Maser, K. and M. Bernhardt, 2000. Statewide bridge deck survey using ground penetrating radar. Proceedings of the Structural Materials Technology IV-An NDT Conference, Feb. 28-Mar. 3, National Academy of Sciences, Atlantic City, New Jersey, 
Am. J. Engg. \& Applied Sci., 4 (4): 440-447, 2011

Rickard, L., 2011. Development of a Procedure for the Detection of Subsurface Defects in Bridge Deck Joint Armor using Ground Penetrating Radar and Seismic Properties Analysis. Ph.D. Thesis, North Carolina A and T State University.

Scotta, M., A Rezaizadeha, A. Delahazab, C.G Santosc and M. Moored et al., 2003. A comparison of nondestructive evaluation methods for bridge deck assessment. NDT Int., 36: 245-255. DOI: 10.1016/S0963-8695(02)00061-0
Xanthakos, P.P., 1996. Bridge Strengthening and Rehabilitation. 1st Edn., Prentice Hall PTR, Upper Saddle River, New Jersey, ISBN: 0133627160, pp: 966.

Young, H.D. and R.A. Freedman, 1999. Sears and Zemansky's University Physics. 10th Edn., Addison-Wesley, San Francisco, ISBN-10: 0201603225, pp: 1274. 